\title{
A Atenção Básica e os cuidados intermediários: um debate necessário
}

Primary Care and intermediate care: a necessary debate

Túlio Batista Franco', Luiz Carlos Hubner ${ }^{\mathbf{1}}$

DOI: 10.1590/0103-1104202012518

RESUMO Este artigo pretende discutir a Atenção Básica, tomando como pressuposto a questão estrutural quanto ao seu escopo de atribuições. Isso tem sido tema de grande debate entre os formuladores de políticas, órgãos reguladores nacionais e internacionais, e constata-se que há uma tendência a considerar a Atenção Básica como um seguimento fundamental e estruturante da rede de serviços de saúde. Isso também significa superar a ideia já muito propagada de que a atenção primária tem foco em serviços de prevenção e promoção, com clínica básica, ofertando cesta reduzida de serviços, com objetivo de respon-der a algumas necessidades de grupos populacionais de baixa renda, sem a devida integralidade do sistema, com acesso a todos os recursos necessários ao cuidado.

PALAVRAS-CHAVE Atenção Primária à Saúde. Gestão em saúde. Cuidados críticos.

\begin{abstract}
This article aims at discussing Primary Care, based on the assumption of the structural question as to its scope of duties. This has been a topic of great debate among policy makers, national and international regulatory bodies, and there is a tendency to consider Primary Care as a fundamental and structuring followup of the health services network. This also means overcoming the already widespread idea that primary care focuses on prevention and promotion services, with basic clinic, offering a reduced package of services, with the aim of meeting some needs of low-income population groups, without proper completeness of the system, with access to all the resources necessary for care.
\end{abstract}

KEYWORDS Primary Health Care. Health management. Critical care. 


\section{Introdução}

Este artigo pretende discutir a Atenção Básica $(\mathrm{AB})$, tomando como pressuposto a questão estrutural quanto ao seu escopo de atribuições. Isso tem sido tema de grande debate entre os formuladores de políticas, órgãos reguladores nacionais e internacionais, e constata-se que há uma tendência a considerar a $\mathrm{AB}$ como uma rede de serviços absolutamente fundamental e estruturante dos sistemas de saúde. Isso significa superar a ideia já muito propagada de que

[...] a atenção primária é interpretada como um programa focalizado e seletivo, que oferta cesta reduzida de serviços, com objetivo de responder a algumas necessidades de grupos populacionais de baixa renda, mesmo sem garantir possibilidade de acesso a outros recursos do sistema1(868).

As concepções atuais têm colocado a $\mathrm{AB}$ em um lugar mais relevante, adotando o conceito de integralidade que traz a importante ideia de que deve haver uma rede de serviços conectada para garantir a oferta de serviços e o acesso, no atendimento às necessidades dos usuários.

[...] a APS [Atenção Primária à Saúde] é o primeiro contato da assistência continuada centrada na pessoa, de forma a satisfazer suas necessidades de saúde, que só refere os casos muito incomuns que exigem atuação mais especializada. A APS coordena, ainda, os cuidados quando as pessoas recebem assistência em outros níveis de atenção. Starfield sugere os seguintes atributos para as práticas da atenção primária: primeiro contato, longitudinalidade, integralidade e coordenação ${ }^{\mathbf{1 ( 8 6 9 )} \text {. }}$

Embora haja uma centralidade maior para a $\mathrm{AB}$ na hora de pensar as políticas e a organização dos serviços de saúde, permanece um escopo de atribuições reduzido com relação aos atributos que poderiam ser imputados à rede básica, especialmente quando se trata de pensar os usuários, por exemplo, em condições crônicas, que venham a ter uma piora na sua situação de saúde ao apresentar um quadro agudo. Pessoas com baixa autonomia e níveis elevados de dependência encontram pouco respaldo na $\mathrm{AB}$ para a continuidade do seu cuidado. Situações como essas revelam seus limites e colocam em questão a sua resolutividade e capacidade de ser a 'ordenadora da rede de serviços de saúde'. Entende-se que desse ponto há uma lacuna a ser preenchida, ou seja, a $\mathrm{AB}$ deveria ter a possibilidade de também dar continuidade ao cuidado nessas situações.

Neste sentido, pretende-se discutir neste texto a necessária expansão do escopo de atribuições da $A B$, supondo um aumento da sua resolutividade, atuando intensamente, também, em problemas de saúde relacionados às condições agudas e de baixa autonomia dos usuários. Nesses termos, pretende-se pensar em uma $\mathrm{AB}$ como uma rede robusta, capaz de ofertar atenção aos usuários desde o cuidado domiciliar e no território até a possibilidade de cuidados intermediários, capaz de atender, também, às necessidades dos subagudos, dos crônicos ou egressos de internação. Tal condição daria à rede básica de saúde uma alta potência na gestão do cuidado e, ao mesmo tempo, deve operar em rede com todo sistema de saúde.

Propõe-se discutir aqui, juntamente com o tema a $\mathrm{AB}$, os cuidados intermediários e, em especial, um dispositivo muito difundido na Europa, os Hospitais Comunitários, cujas caraterísticas e definição serão explicitadas à frente. Este texto propõe empreender uma discussão com base em três dimensões do problema, especialmente com foco na micropolítica da gestão e do trabalho em saúde. São elas:

1. A possibilidade de qualificação e expansão dos cuidados aos crônicos, contribuindo para isso os vários dispositivos de possibilidades, inclusive a ideia de 'Hospital Comunitário', nos termos do NHS (National Health Service) da Inglaterra ${ }^{2}$, e tomando como subsídio também a experiência dos serviços de saúde da Itália e de outros países europeus. Esse equipamento estaria vinculado à $\mathrm{AB}$ como 
mais uma instância de trabalho e ferramenta para o cuidado, atuando em rede, com uso massivo de tecnologias leves nos processos de trabalho.

2. Aumento do escopo de atuação da $\mathrm{AB}$ no que se refere ao cuidado à saúde. Para tanto, propõe-se utilizar os cuidados intermediários, aqueles que se situam entre a $\mathrm{AB}$ e a hospitalar.

3. A formação de uma rede operando sob a gestão do cuidado que garanta a formação das linhas de cuidado, especialmente a usuários portadores de condições crônicas ou egressos de internação hospitalar e que necessitam de ganhos de autonomia.

Para fazer esta discussão, procurou-se debater o tema referente à construção da $\mathrm{AB}$, entendendo-a como uma instância de cuidado estratégica para o ordenamento da rede de saúde. É por considerar tal perspectiva que será considerado, no decorrer desta discussão, que a $A B$ também incorpore os cuidados intermediários.

É fácil constatar que desde o movimento da reforma sanitária, na década de 1970, até os dias atuais, a $\mathrm{AB}$ tem sido o centro do discurso reformista e de construção do SUS. Esperavase com a organização de uma pujante rede básica uma grande revolução, que passava pela inversão dos gastos do sistema hospitalar e da rede de média e alta complexidade para a atenção básica. Ao mesmo tempo, apostou-se fortemente na descentralização dos serviços de saúde para os municípios. Combinado a isso, sempre se perseguiu a ideia de que seria possível resolver em torno de $85 \%$ dos problemas de saúde da população com uma rede básica bem montada, equipada e pessoal treinado.

Após a consolidação do Sistema Único de Saúde (SUS), com a Lei Orgânica da Saúde, aprovada em 1990, houve uma reorganização do modo de organização dos serviços, com a proposta do Ministério da Saúde (MS) de implantar o Programa de Saúde da Família, em 1994, que se transformou na Estratégia Saúde da Família (ESF), em 2003. A nova modelagem resultou em um forte investimento de recursos na $\mathrm{AB}$.

No ano de 2004, o PSF abrangia 80,2\% dos municípios brasileiros, 4.600 municípios, proporcionando uma cobertura de $39 \%$ da população, o que correspondia a 69,1 milhões de pessoas. Quando publicada a nova Política Nacional de Atenção Básica (PNAB), no ano de 2006, foram implantadas 26.729 Equipes de Saúde da Família (EqSF), em 5.106 municípios, cobrindo $46,2 \%$ da população brasileira, o que correspondia a cerca de 85,7 milhões de pessoas. Em síntese, ao longo de todo o período, o número de EqSF, 328, em 1994, ultrapassou 33 mil em 2012, atingindo uma cobertura da população brasileira (193.946.886) de $54,8 \%{ }^{3}$.

A expansão da ESF ganhou ritmo acelerado nesse período específico, sendo que no

[...] final de 2013, constatou-se a presença de 36 mil equipes da Estratégia Saúde da Família (ESF), de 300 mil Agentes Comunitários de Saúde e de 23 mil equipes de Saúde Bucal (EqSB) ${ }^{3(901) . ~}$

Ao mesmo tempo que houve uma mudança na organização dos serviços, o MS aumentou o repasse para a $\mathrm{AB}$ de forma substancial até o ano de 2014, como nos informa estudo feito pelo Instituto de Pesquisa Econômica Aplicada (Ipea). De acordo com o Ipea, os recursos destinados à $\mathrm{AB}$ passaram de $9,7 \%$ para $17,7 \%$, no período de 1995 a $2011^{6}$. Isso foi decorrente da política prioritária do MS de fortalecimento das ações ou programas da $\mathrm{AB}$, especialmente os incentivos financeiros para o Programa Saúde da Família. Os recursos destinados especificamente para o Programa de Agentes Comunitários de Saúde evoluíram de 0,6\% do total, em 1995, para 8,9\%, em $2009^{4}$.

Podemos constatar que houve no Brasil, nesse período, um importante investimento na AB. Embora isso tenha sido muito positivo, com efeitos importantes na sua expansão, observa-se que não foi suficiente em relação 
à aposta que se fez, de ver a rede básica como principal referência do sistema de saúde, ordenadora da rede de cuidado e com alta resolutividade, fazendo a gestão das redes que se conectam para o cuidado e constituindo linhas de cuidado no emaranhado da rede SUS. A questão que mais intriga nesse cenário é o fato de que, após vários anos de importante investimento, a $\mathrm{AB}$ não alcança o objetivo proposto de ser a real condutora dos processos de cuidado. Há algum problema estrutural que impede o desenvolvimento da rede básica, para que atinja o objetivo pensado na sua fundação, e que persiste ainda hoje, após todos esses anos desde a constituição do SUS.

No entendimento dos autores, identificaram-se duas questões que podem estar impedindo a $\mathrm{AB}$ de assumir sua principalidade na rede de serviços do SUS:

$1^{\circ}$. Mesmo que tenha havido uma reorganização dos serviços de $\mathrm{AB}$, com a instituição do Programa ou Estratégia Saúde da Família, essa mudança aconteceu apenas na estrutura de organização das equipes de trabalho, com baixo impacto no processo de trabalho. Este se limita a alterar o centro de gravidade da produção do cuidado da unidade de saúde para o território e o domicílio, prioritariamente. Mesmo que essa mudança seja importante, representa uma reestruturação produtiva, ou seja, muda o modo de produzir o cuidado. Ela é insuficiente para transformar a essência do trabalho, o seu núcleo tecnológico. Tais limites indicam, principalmente, que as diretrizes de reordenamento do processo de trabalho da ESF não conseguem orientar para que as equipes invertam seu processo de trabalho, mudando a centralidade das tecnologias de cuidado, com maior densidade das tecnologias leves no processo de trabalho 5 . Isso efetivamente não era tratado pelo MS, e, portanto, o próprio PSF continuava operando principalmente no modelo 'produtor de procedimentos', mesmo atendendo as pessoas no domicílio e, assim, operando da mesma forma o cuidado com base no alto consumo de tecnologias duras, em detrimento das leves. Por essa razão, os fluxos produtivos do cuidado continuavam fortemente voltados à alta e à média complexidade e ao sistema hospitalar, mesmo que aqueles tenham reduzido uma pequena margem no seu financiamento federal em relação à $\mathrm{AB}$.

$2^{\circ}$. O escopo da $\mathrm{AB}$ permanece restrito e limitado a processos de prevenção e promoção da saúde, com a intervenção clínica, que ampliou em muitos lugares sua atuação, no entanto, ela permanece restrita na medida em que não há recursos suficientes para o atendimento ao agudo ou a pessoas com baixa autonomia, em processos nos quais seriam necessárias ações de recuperação e reabilitação. A discussão de cuidados intermediários problematiza o atual escopo de atribuições da $\mathrm{AB}$. O que se discute, com base nas experiências internacionais, e mesmo na experiência brasileira, é a vinculação das ações de cuidado intermediário à $\mathrm{AB}$, o que não significa um vínculo administrativo apenas, e às vezes nem isso seria adequado em muitos casos, mas uma conexão com fluxos seguros em rede, coordenado pela $\mathrm{AB}$, ou seja, ela seria a gestora do cuidado, incluindo os cuidados intermediários na sua governabilidade. A AB com eficácia no cuidado ao agudo, e mesmo em situações de baixa autonomia, é a urgência do momento, especialmente ao se verificar o sucessivo crescimento da população idosa em relação à população geral, nas últimas décadas, que será discutido mais à frente. Assim, torna-se necessária uma reconfiguração dos serviços de saúde no Brasil, para atender às novas e importantes demandas dos serviços, principalmente aos cuidados aos crônicos e egressos de internação hospitalar, necessitando da continuidade do cuidado.

$3^{\circ}$. A gestão do cuidado tem como pressuposto a constituição de redes que operam a partir do trabalho cotidiano, onde os trabalhadores 
fazem os fluxos assistenciais acontecerem, e operam em conexão entre eles próprios e com os usuários. Nesse sentido, sugere-se observar que a micropolítica do processo de trabalho tem sido a referência importante para análise do funcionamento das linhas de cuidado. Essas linhas que refletem o percurso do usuário na rede de saúde são a expressão material e mais intensa dessas redes, mesmo que as redes normativas e protocolares tenham seu destaque como referência para esses fluxos. Entre as redes que regulam e as que são criadas no processo de trabalho cotidiano, há uma tensão constitutiva desses cenários, que são parte intrínseca do funcionamento dessas redes, porque são sempre o produto da ação dos trabalhadores.

Historicamente, os serviços de saúde no Brasil se organizaram de forma polar, ou seja, de um lado, a AB, e, de outro, a hospitalar. Não há entre ambos um serviço amplo, consistente, em larga escala, constituído como uma rede, com cuidados intermediários, que serviriam como uma instância de cuidado que poderia trabalhar no âmbito da recuperação e da reabilitação, cuidando de pessoas com sinais de agudização, em estado agudo e egressos de internação, ainda com baixa autonomia. A missão desses serviços, e mesmo os de uma Unidade de Cuidados Intermediários, seria a de elevar o grau de autonomia do usuário para que este retorne em segurança para o domicílio.

Viu-se até aqui que, no Brasil, vivemos um paradoxo que constata ter havido muito investimento na $A B$, especialmente na primeira década e meia deste século. São investimentos materiais que significam expansão territorial da cobertura, construção e reformas de prédios, assim como aumento de equipes; e em recursos imateriais, como a educação permanente e os programas de formação técnica dos trabalhadores. No entanto, mesmo com altas possibilidades de atender às necessidades de saúde nas comunidades onde de instala, há uma lacuna na atenção ao agudo e a pessoas com baixa autonomia. Ao que parece, a rede básica chegou ao seu limite de possibilidades com relação ao atual escopo de atribuições, contando com os recursos assistenciais de que dispõe. Essa parece a principal discussão a ser tratada, quando se fala em cuidados intermediários vinculados à $\mathrm{AB}$.

\section{Revendo a experiência de cuidados intermediários no Brasil}

O fortalecimento da $\mathrm{AB}$, sobretudo para o cuidado aos crônicos, coloca-se desde sempre como um fator importante para qualidade dos serviços de saúde. Hoje é uma emergência, especialmente diante do fenômeno de altas taxas de crescimento da população idosa, em razão da população geral no Brasil. Essa população mais susceptível às condições crônicas precisa encontrar respostas consistentes das redes de atenção à saúde, especialmente na $\mathrm{AB}$, para o atendimento às suas necessidades. Assim, serviços com capacidade de operar com foco na recuperação e na reabilitação, especialmente fazendo uso central das tecnologias leves, no processo de produção do cuidado, são fundamentais para dar essa resposta.

Verificando o cenário demográfico dos últimos anos, dados do Instituto Brasileiro de Geografia e Estatística (IBGE) informam que a população brasileira acima de 60 anos, no ano de 2.000 , representava $8,2 \%$ do total; em 2017, são $12,5 \%$; e projeta-se para o ano de 2030 um percentual de 18,6\%. Já acima de 50 anos, hoje são $23,3 \%$, e em 2030 serão $31,2 \%$ da população. Nota-se a importância de ter uma política eficaz no cuidado ao idoso, em especial, às doenças crônicas. Uma importante alternativa aos altos custos hospitalares e a um cuidado de melhor qualidade são os cuidados intermediários, que se situam entre a AB e a hospitalar. No Brasil, apenas recentemente teve regulamentação governamental, através da Portaria ${ }^{\circ} 895$, de 31 de março de $2017^{6}$. 
O MS do Brasil informa que, no ano de 2016, foram realizadas 11.282 .302 internações hospitalares, sendo $7,1 \%$ de alta complexidade, e $92,9 \%$ de média complexidade, conforme classificação do MS. Os gastos correspondentes foram de $\mathrm{R} \$ 11.145 .926 .740,99$, dos quais, $30,6 \%$ na Alta Complexidade e $69,4 \%$ na Média Complexidade7. Supõe-se que parte das internações de média complexidade poderia ser evitada se houvesse uma instância de cuidados intermediários, situada entre a $\mathrm{AB}$ e a hospitalar, capaz de ações de recuperação e reabilitação. Tal medida, além de produzir um cuidado mais eficaz, possivelmente reduziria custos ao sistema de saúde.

Uma melhor resolutividade na $\mathrm{AB}$ pode, efetivamente, reduzir internações desnecessárias e inadequadas, otimizando o uso de leitos, com possibilidade de aumentar essa oferta. Esse texto propõe uma discussão para sistematizar e analisar as experiências de cuidados intermediários existentes junto à $\mathrm{AB}$ no Brasil e em países parceiros europeus, especialmente vinculados a essa proposta. Os resultados da discussão devem servir para um debate em torno da ideia de incremento de ações e serviços junto à rede básica.

Os cuidados intermediários, tal como sugeridos aqui, os que se situam entre $\mathrm{AB}$ e a hospitalar, podem servir para atender a uma demanda de problemas de saúde relacionada a casos em que o usuário tem necessidades de cuidado que estão acima das condições às quais a $\mathrm{AB}$ consegue atender, mas que, por outro lado, não se enquadram no perfil para a assistência hospitalar. Sendo assim, eles são capazes de oferecer um cuidado mais adequado a esses usuários e, ao mesmo tempo, prevenir internações desnecessárias, trabalhando a recuperação e a reabilitação do crônico ou da pessoa com baixa autonomia.

O termo 'cuidados intermediários', no Brasil, tem sido usado para nomear os serviços que estão atrelados à linha de cuidado progressivo ao paciente crítico ou grave, conforme Portaria do MS n ${ }^{\circ} 895 / 2017$. Nessa portaria, os cuidados intermediários se situam entre a unidade de internação e a Unidade de Terapia Intensiva:

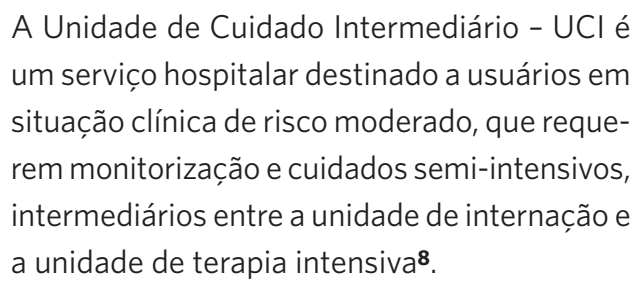

Para efeito deste artigo, cuidados intermediários são entendidos como aqueles que se situam entre a $\mathrm{AB}$ e o hospital, destinados a usuários em situação crônica e em processo de agudização, ou com baixa autonomia, necessitando de cuidados de reabilitação ou recuperação, para os quais os recursos disponibilizados em domicílio ou na $\mathrm{AB}$ são insuficientes. Nesses casos, seria inadequada e desnecessária uma internação em Hospital, local que deve ser reservado para internações em casos que necessitem de tecnologias hospitalares para o seu cuidado.

Os serviços de cuidados intermediários começam a ser definidos nos anos 1990, prevalecendo o que propõe a Sociedade Britânica de Geriatria, segundo a qual tais serviços devem atender a algumas condições, que seriam: 
Quadro 1. Critérios para definição de cuidados intermediários

\begin{tabular}{|c|c|c|c|c|}
\hline $\begin{array}{l}\text { Serviços destinados } \\
\text { àqueles indivíduos } \\
\text { que necessitam de } \\
\text { longa internação } \\
\text { hospitalar, ou com } \\
\text { risco de internação } \\
\text { hospitalar, ou neces- } \\
\text { sidade de assistência } \\
\text { em residências }\end{array}$ & $\begin{array}{l}\text { Serviços articulados } \\
\text { sobre abordagem } \\
\text { global destinada } \\
\text { a desenvolver um } \\
\text { percurso de cuidados } \\
\text { individuais (PAI) }\end{array}$ & $\begin{array}{l}\text { Serviços com o ob- } \\
\text { jetivo principal de } \\
\text { máxima recuperação } \\
\text { funcional e de retorno } \\
\text { ao domicílio }\end{array}$ & $\begin{array}{l}\text { Serviços prestados } \\
\text { por um período nor- } \\
\text { malmente não supe- } \\
\text { rior a } 6 \text { semanas }\end{array}$ & $\begin{array}{l}\text { Serviços que adotem } \\
\text { o método de trabalho } \\
\text { interprofissional com } \\
\text { um um processo } \\
\text { avaliativo baseado em } \\
\text { protocolos comparti- } \\
\text { Ihados }\end{array}$ \\
\hline \multicolumn{5}{|c|}{ PAI - Plano Individual de Cuidado } \\
\hline
\end{tabular}

Fonte: Le Cure Intermedie. Italian Journal of Medicine $\mathbf{8}^{\mathbf{.}}$

Ainda segundo os autores, no âmbito da rede de serviços, algumas das formas possíveis e mais utilizadas de cuidados intermediários são: internação domiciliar; pós-agudização e cuidados de longa duração; Hospital Dia; cuidado de enfermagem domiciliar (residência sanitária e assistencial, casa protegida); Hospital Comunitário.

No Brasil, há vários Serviços de Cuidados Intermediários que têm uma função importante, embora seja necessário maior investimento e a sua ampliação. Alguns exemplos são os Serviços de Atenção Domiciliar (SAD), 'Melhor em Casa', as unidades e os hospitais de cuidados prolongados ${ }^{9}$, que se enquadram no perfil de cuidados intermediários citado acima. Apenas recentemente houve regulamentação governamental de cuidados prolongados e de SAD, estruturas equivalentes aos cuidados intermediários, conforme conceito apresentado aqui.

No Brasil, especificamente, o SAD tem como objetivos: a redução da demanda por atendimento hospitalar ou a redução do período de permanência de usuários internados; a humanização da atenção à saúde, com a ampliação da autonomia dos usuários, a desinstitucionalização e a otimização dos recursos financeiros e estruturais da Rede de Atenção à Saúde (RAS). É organizado por meio das Equipes Multiprofissionais de Atenção Domiciliar (Emad) e Equipes Multiprofissionais de Apoio (Emap), devendo atuar de forma complementar aos cuidados realizados na $\mathrm{AB}$, em serviços de urgência e sendo substitutivo ou complementar à internação hospitalar. Foi lançado, em agosto de 2011, por meio da Portaria GM/MS $\mathrm{n}^{\circ} 2.029$, e atualmente é regulamentado pelas Portarias Consolidadas GM/MS n ${ }^{\circ} 5$ e $n^{\circ} 6$, de 28 de setembro de 2017, tendo abrangência nacional, contando com aproximadamente 1.000 equipes que cobrem cerca de $26 \%$ da população brasileira. Entre os principais perfis atendidos pelo programa estão os pacientes com sequelas de Acidente Vascular Cerebral (AVC), já estabilizados, mas ainda com necessidade intensiva de cuidados, os pacientes com sequelas de traumas (acidentes de trânsito e vítimas de violência) e os pacientes com doenças degenerativas ou ameaçadoras da continuidade da vida, especialmente aqueles em fase final de vida.

\section{Experiências de cuidados intermediários e Hospital Comunitário}

Uma tese da Universidade de Bolonha, Itália, informa que o conceito de 'cuidado intermediário’ nasce na Inglaterra, no ano 2000, no National Beds Enquiry ${ }^{10}$. Discussões sobre esse tema aconteciam desde os anos 1990, e neste século o conceito entra definitivamente na política sanitária europeia. Segundo o autor, o objetivo desses serviços é o de evitar 
internações desnecessárias ou inadequadas, atuando preventivamente no cuidado a usuários antes que se tornem agudos, ou nos cuidados a egressos de internação hospitalar, para que obtenham ganhos de autonomia para o retorno seguro ao seu domicílio.

Importa informar que, como já foi dito anteriormente, há muitos tipos de cuidados intermediários. Neste momento, passa-se a discutir principalmente o Hospital Comunitário, tratando-o como uma novidade que se coloca frente ao SUS, e suas necessidades e possibilidades de cuidado aos usuários. As atuais condições sociossanitárias do Brasil, especialmente com o crescimento da prevalência das condições crônicas, em razão da mudança do perfil censitário da população, indicam aumento da população idosa em relação à população geral do País. Tal situação nos coloca à frente da urgência de uma reestruturação produtiva no segmento da saúde, onde uma nova configuração das redes de atenção deve melhor responder às necessidades de saúde deste momento.

O Hospital Comunitário é uma Unidade de Cuidados Intermediários, situando-se, portanto, entre a $\mathrm{AB}$ e a hospitalar. Serve para internação de usuários com baixa autonomia ou em intercorrência da sua condição crônica. Nos protocolos ingleses e italianos, por exemplo, o período de internação em uma Unidade dessa deve estar dentro do período de até 6 semanas, podendo variar de acordo com a região ou o país. Muito difundido na Europa atualmente, ele tem sido um importante dispositivo na melhora do cuidado, especialmente ao crônico, ou egressos do hospital, mas ainda com baixa autonomia. Contribui de forma importante para o aumento da resolutividade da $A B$ e é especialmente eficiente na relação custo-benefício, por incidir sobre a redução de internações desnecessárias e inadequadas em Hospitais Gerais.

Em exame no relatório de auditoria realizada sobre os serviços de cuidados intermediários, publicado no 'National Audit of Intermediate Care Summary Report 2015’, na
Inglaterra, verificou-se que 70\% dos usuários que recebem alta do Hospital Comunitário são encaminhados ao domicílio, revelando uma alta resolutividade, ou seja, demonstra que o objetivo de evitar internações no Hospital Geral está sendo cumprido. Outros 10\% apenas vão para um Hospital Geral para agudos; e 8\% para recuperação em casa, aproximadamente"1.

Na mesma direção do informe inglês, a eficácia do Hospital Comunitário no cuidado é comprovada em estudo realizado na Noruega, que comparou pacientes internados no Hospital Comunitário com aqueles do Hospital Geral, e conclui que:

O cuidado intermediário em um Hospital Comunitário diminuiu significativamente $\mathrm{O}$ número de readmissões para a mesma doença para o hospital geral e um número significativamente maior de pacientes ficou independente do atendimento comunitário após 26 semanas de seguimento, sem aumento na mortalidade e número de dias em instituições ${ }^{\mathbf{1 2 ( 8 )} \text {. }}$

Ainda segundo esse estudo:

Os cuidados intermediários em um Hospital Comunitário parecem ser altamente efetivos. Em um sistema de cuidados de saúde moderno, o cuidado é cada vez mais especializado, fragmentado e focado em órgãos. Além da expansão de uma sub-especialização adicional na medicina moderna, os resultados deste estudo ressaltam a necessidade adicional de melhores sistemas de cuidados de step-down em um nível intermediário 12(7).

A Itália e, em especial, a Região EmiliaRomagna têm feito investimentos significativos na organização de serviços de atenção intermediária, incluindo o Hospital Comunitário.

Um informe da Região registra um quadro da rede de serviços da cidade de Modena, no qual é possível observar muitos pontos de convergência com situações vivenciadas nos serviços de saúde no Brasil: 1) 'o fenômeno do envelhecimento da população’ e 
o consequente aumento de incidência de doenças crônicas, que necessitam de políticas fortemente voltadas para esse cuidado; 2 ) 'a fragmentação do cuidado a essa população', resultando em dificuldades na assistência e risco de piora no quadro clínico; 3) 'internações hospitalares desnecessárias e inapropriadas', piorando as condições de cuidado do usuário e elevando a relação custo-efetividade na prestação de serviços de saúde; 4) 'a insuficiência na rede de serviços de saúde de estruturas para cuidados intermediários' destinadas ao usuário crônico ou ao egresso de internação hospitalar e que necessita de suporte para retornar para casa ${ }^{13}$.

O Plano Social e Sanitário de 2017-2019 da Região de Emilia-Romagna, Itália, define o Hospital Comunitário da seguinte forma:

uma estrutura com número limitado de leitos, gestão da enfermagem, e assistência médica é garantia pelo Médico de Medicina Geral, o Pediatra de livre escolha ou o Médico Servidor Público do Serviço Sanitário Nacional. Atende prevalentemente pacientes que necessitam de acompanhamento de enfermagem de forma continuada, ou de cuidados potencialmente ofertados em domicílio, mas que necessitam de internação nesta estrutura, na falta de estrutura e acompanhamento familiar no domicílio ${ }^{14(37)}$.

Há um investimento importante da Região na organização de Hospitais Comunitários. $\mathrm{O}$ documento citado acima informa que existiam, em 2017, 13 Hospitais Comunitários, com um total de 230 leitos, exercendo atividades de cuidados intermediários em Emilia-Romagna.

Pela história e pela experiência no National Health Service inglês, na Noruega e na Itália, discutidas acima, é possível formar evidência quanto à eficácia e eficiência desses serviços, podendo servir de referência para se pensar um projeto de ampliação e qualificação dos cuidados intermediários junto ao SUS no Brasil, particularmente, pensando a implantação de Unidades de Cuidados Intermediários vinculadas à $\mathrm{AB}$.
Todas essas questões de reorganização dos serviços de saúde devem ter como pressuposto uma importante intervenção na micropolítica do processo de trabalho, essência do modo de produção do cuidado ${ }^{15}$. Propõe-se pensar a questão também nessa perspectiva.

\section{Considerações finais}

Este texto pretendeu levantar um debate, e não fechá-lo aqui, que procura problematizar a AB, sobretudo a abrangência atual do seu escopo de atribuições, propondo que o mesmo seja estendido para abarcar um volume maior de atividades e dispositivos para o cuidado à saúde.

Os cuidados intermediários foram pensados para isso, tal como exposto aqui, como dispositivos de cuidado que se situam entre a $\mathrm{AB}$ e a hospitalar, fazendo mediação entre tais serviços, através de uma potente rede de cuidado, com fluxos seguros aos usuários. Especialmente a vinculação destes serviços com a $\mathrm{AB}$ parece a questão importante, pois significa uma reconfiguração da rede de serviços, em especial, o fortalecimento dos cuidados no âmbito local e das comunidades.

Espera-se, com isso, aumentar a resolutividade no cuidado aos crônicos, demanda cada vez mais frequente nas redes de serviços, e que deve ser prioridade, sobretudo com o fenômeno da transição demográfica, com o aumento significativo da população idosa em relação à população geral, aumentando a demanda para esse perfil de usuário.

Por outro lado, o Brasil traz uma importante experiência de cuidados, inscrita no Programa de Atenção Domiciliar (PAD), e os cuidados prolongados, que têm vários dispositivos assistenciais. Esses programas, embora significativos e regulamentados, ainda são insuficientes para atender à demanda existente e certamente crescente.

Espera-se ter suscitado com este artigo o debate sobre o tema que envolve o fortalecimento da $\mathrm{AB}$ e as mudanças estruturais na 
forma de pensar e fazer os serviços de saúde em que a questão do seu funcionamento cotidiano esteja em foco. Espera-se contribuir para inovações no campo da produção do cuidado, inserindo no debate da $\mathrm{AB}$ o dispositivo de cuidados intermediários como um elemento de mudança no rumo da sua qualificação e ampliação de possibilidades de cuidado.

\section{Referências}

1. Lavras C. Atenção primária à saúde e a organização de redes regionais de atenção à saúde no Brasil. Saúde soc. [internet]. 2011 [acesso em 2020 maio 20]; 20(4):867-874.

2. NHS Benchmarking Network. National Audit of Intermediate Care: summary report 2015. Assessing progress in services for older people aimed at maximising independence and reducing use of hospitals. Social Care [internet]. 2015. [acesso em 2020 maio 30]. Disponível em: http://www.scie-socialcareonline.org. uk/national-audit-of-intermediate-care-summary-report-2015-assessing-progress-in-services-for-older-people-aimed-at-maximising-independence-and-reducing-use-of-hospitals/r/allG000000CTEgOIAX.

3. Mendes A, Marques RM. O financiamento da Atenção Básica e da Estratégia Saúde da Família no Sistema Único de Saúde. Saúde debate. 2014; 38(103):900916.

4. Servo LMS, Piola SF, Sá EB, et al. Brasil em desenvolvimento 2011: Estado, planejamento e políticas públicas. Ipea: Brasília, DF; 2011.

\section{Colaboradores}

Franco TB (0000-0001-7372-5262)* e Hubner LC (0000-0001-7073-9888)* contribuíram igualmente para a elaboração do manuscrito.
5. Franco TB, Bueno WS, Merhy EE. O acolhimento e os processos de trabalho em saúde: o caso de Betim, Minas Gerais, Brasil. Cad. Saúde Pública [internet]. 1999 [acesso em 2020 maio 20]; 115(2):345-353. Disponível em: https://www.scielo.br/scielo.php?pid=S010 2311X1999000200019\&script=sci_abstract\&tlng=pt.

6. Brasil. Ministério da Saúde. Portaria ${ }^{\circ} 895$, de 31 de março de 2017. Institui o cuidado progressivo ao paciente crítico ou grave com os critérios de elegibilidade para admissão e alta, de classificação e de habilitação de leitos de Terapia Intensiva Adulto, Pediátrico, Unidade Coronariana, Queimados e Cuidados Intermediários Adulto e Pediátrico no âmbito do Sistema Único de Saúde (SUS). Diário Oficial da União. 2017 Mar 31. [acesso em 2020 jun 2]. Disponível em: https://bvsms.saude.gov.br/bvs/saudelegis/gm/2017/ prt0895_26_04_2017.html.

7. Brasil. Ministério da Saúde. DATASUS. [acesso em 2017 ago 15]. Disponível em: www.datasus.gov.br.

8. Salsi A, Calogero P. Le Cure Intermedie. Italian J. Med. 2010 [acesso em 2020 jun 9]; 4:57-62. Disponí-
*Orcid (Open Researcher and Contributor ID). 
vel em: https://www.italjmed.org/index.php/ijm/article/view/itjm.2010.57/253.

9. Brasil. Ministério da Saúde. Portaria n ${ }^{0} 2.809$, de 7 de dezembro de 2012. Estabelece a organização dos Cuidados Prolongados para retaguarda à Rede de Atenção às Urgências e Emergências (RUE) e às demais Redes Temáticas de Atenção à Saúde no âmbito do Sistema Único de Saúde (SUS). Diário Oficial da União. 7 Dez 2012. [acesso em 2020 maio 20]. Disponível em: http://bvsms.saude.gov.br/bvs/saudelegis/ gm/2012/prt2809_07_12_2012.html.

10. Pieri G. L'Ospedale di Comunità nella Regione Emilia-Romagna: modelli organizzativi e valutazione della qualità dell'assistenza. [tese]. Bologna: Universidade de Bologna; 2016.

11. NHS Benchmarking Network. National Audit of Intermediate Care: summary report 2015. Assessing progress in services for older people aimed at maximising independence and reducing use of hospitals. 2015. [acesso em 2017 set 10]. Disponível em: http://www. scie-socialcareonline.org.uk/national-audit-of-intermediate-care-summary-report-2015-assessing-progress-in-services-for-older-people-aimed-at-maximising-independence-and-reducing-use-of-hospitals/r/ allG000000CTEgOIAX.
12. Garåsen H, Windspoll R, Johnsen R. Intermediate care at a community hospital as an alternative to prolonged general hospital care for elderly patients: a randomised controlled trial. BMC Public Health. 2007 [acesso em 2019 set 20]; 7:68. Disponível em: https://www.ncbi.nlm.nih.gov/pmc/articles/PMC1 868721/\#idm140430022376160title.

13. Servizio Assistenza Territoriale. Direzione Generale Cura della Persona, Salute e WelfareUm novo avanço no desenvolvimento da rede de serviços de assistência territorial e a implantação dos Hospitais Comunitários na Região Emilia-Romagna. Emilia-Romagna: RER; 2016.

14. Itália. Região Emilia-Romagna. Plano Social e Sanitário de 2017-2019 da Região de Emilia-Romagna: Ministério da Saúde; 2019.

15. Franco TB, Merhy EE. Trabalho, Produção do Cuidado e Subjetividade em Saúde. São Paulo: Hucitec; 2013.

Recebido em 01/10/2019

Aprovado em 05/05/2020

Conflito de interesses: inexistente

Suporte financeiro: não houve 